\title{
Two Isoperimetric Inequalities for the Sobolev Constant*
}

\author{
Tom Carroll and Jesse Ratzkin \\ University College Cork and University of Cape Town \\ t.carroll@ucc.ie and jesse.ratzkin@uct.ac.za
}

September 26, 2018

\begin{abstract}
In this note we prove two isoperimetric inequalities for the sharp constant in the Sobolev embedding and its associated extremal function. The first such inequality is a variation on the classical Schwarz Lemma from complex analysis, similar to recent inequalities of Burckel, Marshall, Minda, Poggi-Corradini, and Ransford, while the second generalises an isoperimetric inequality for the first eigenfunction of the Laplacian due to Payne and Rayner.
\end{abstract}

\section{Introduction and statement of results}

Let $p \geq 1$ and let $D$ be a bounded domain in the plane, equipped with the usual Lebesque area measure $d A$. The well-known Sobolev embedding theorem states that there exists a constant $k=k(p, D)$ such that

$$
u \in W_{0}^{1,2}(D) \Rightarrow\|u\|_{L^{p}(D)} \leq k\|\nabla u\|_{L^{2}(D)},
$$

so that $W_{0}^{1,2}(D) \hookrightarrow L^{p}(D)$. (This embedding is always compact for finite $p$ in two dimensions.) The sharp constant in the Sobolev embedding,

$$
\mathcal{S}_{p}(D)=\sup \left\{\frac{\left(\int_{D} u^{p} d A\right)^{1 / p}}{\left(\int_{D}|\nabla u|^{2} d A\right)^{1 / 2}}: u \in W_{0}^{1,2}(D), u \not \equiv 0\right\},
$$

\footnotetext{
*AMS subject classification: $35 \mathrm{~J}$
} 
is an important and well-studied constant related to the geometry and function theory of $D$. For example, van den Berg [2] has recently derived asymptotic expansions for $\mathcal{S}_{p}(D)$ as $p$ tends to $\infty$. For our purposes, it is slightly more convenient to work with

$$
\mathcal{C}_{p}(D)=\left(\mathcal{S}_{p}(D)\right)^{-2}=\inf \left\{\frac{\int_{D}|\nabla u|^{2} d A}{\left(\int_{D} u^{p} d A\right)^{2 / p}}: u \in W_{0}^{1,2}(D), u \geq 0, u \not \equiv 0\right\} .
$$

The sharp Sobolev constant $\mathcal{S}_{p}$ and its associated extremal functions are the subject of a vast literature which we make no attempt to survey here. Suffice to say that they are still the subject of much intense research and, in particular, there is a well-established link between (1.1) and the isoperimetric inequality (see [8] and references therein).

Sobolev constants appear in a different guise in the theory of elasticity and the theory of vibrating membranes. The constant $\mathcal{C}_{2}(D)$ minimises the Rayleigh-Ritz quotient and corresponds to the first Dirichlet eigenvalue $\lambda(D)$ of the Laplacian for $D$ (or, more generally, the bottom of the spectrum). The constant $\mathcal{C}_{1}(D)$ is related to the torsional rigidity $P(D)$ by $\mathcal{C}_{1}(D)=4 / P(D)$. In this sense, $\mathcal{C}_{p}(D)$ interpolates between the torsional rigidity of a domain and its principal frequency as $p$ ranges from 1 to 2 .

By the compactness of the embedding $W_{0}^{1,2}(D) \hookrightarrow L^{p}(D)$, a minimiser $\phi$ of the ratio defining $\mathcal{C}_{p}(D)$ exists. The Euler-Lagrange equation tells us that $\phi$ solves the boundary value problem

$$
\Delta \phi+\Lambda \phi^{p-1}=0,\left.\quad \phi\right|_{\partial D}=0,
$$

and an integration by parts agrument ([5, Lemma 2]) shows that $\Lambda$ and $\mathcal{C}_{p}(D)$ are related by

$$
\mathcal{C}_{p}(D)=\Lambda\left(\int_{D} \phi^{p} d A\right)^{(p-2) / p}
$$

Our aim in this paper is two-fold: to prove a reverse Hölder inequality for the minimiser in (1.3) which generalises the inequality of Payne and Rayner [14] for the first Dirichlet eigenfunction of the Laplacian and, second, to prove a Schwarz Lemma for the constant $\mathcal{C}_{p}(D)$. In the rest of this introduction, we describe each of these inequalities in detail, and discuss how each is an isoperimetric inequality.

\subsection{A variation on the Schwarz Lemma}

Let $f$ be a complex analytic function in the unit disc $\mathbb{D}=\{z:|z|<1\}$ in the complex plane $\mathbb{C}$ with $f(0)=0$. Let $M(r)=\max \{|f(z)|:|z|=$ 
$r$ \}, $0<r<1$, be the maximum modulus of $f$ on the circle of radius $r$. Then $\lim _{r \rightarrow 0+} M(r) / r=\left|f^{\prime}(0)\right|$ and $\lim _{r \rightarrow 1-} M(r) / r=\|f\|_{\infty}$. The classical Schwarz Lemma states that $M(r) / r$ increases with $r$ and that, if $M(r) / r$ takes the same value at two distinct $r \in[0,1]$, then $f$ is linear.

Burckel, Marshall, Minda, Poggi-Corradini, and Ransford [4] recently proved versions of the Schwarz Lemma for diameter, logarithmic capacity, and area. Laugesen and Morpurgo [11] proved a version of Schwarz's Lemma for principal frequency, in fact for the sum of the values of a convex increasing function applied to the reciprocals of the first $n$ Dirichlet eigenvalues of the Laplacian. More recently, Betsakos has obtained counterparts of the results of Burckel et al. for quasiregular mappings [3]. We adapt the method of Laugesen and Morpurgo to prove a version of Schwarz's lemma for $\mathcal{C}_{p}$.

Theorem 1. Let $f$ be a conformal mapping of the unit disk $\mathbb{D}$ and let $p \geq 1$. The function

$$
r \mapsto \frac{\mathcal{C}_{p}(f(r \mathbb{D}))}{\mathcal{C}_{p}(r \mathbb{D})}=\frac{r^{4 / p}}{\mathcal{C}_{p}(\mathbb{D})} \mathcal{C}_{p}(f(r \mathbb{D})), \quad 0<r<1,
$$

is strictly decreasing unless $f$ is linear (in which case this function is constant). Moreover, if $p \leq 2$, the reciprocal of this function is a convex function of $\log r$.

The simple scaling law, $\mathcal{C}_{p}(r D)=r^{-4 / p} \mathcal{C}_{p}(D)$, was used in (1.6). Using monotonicity and taking the limit of the right hand side of (1.6) as $r \rightarrow 0^{+}$, we obtain

$$
\mathcal{C}_{p}(f(\mathbb{D})) \leq \mathcal{C}_{p}(\mathbb{D})\left|f^{\prime}(0)\right|^{-4 / p},
$$

which generalises the eigenvalue estimate in Section 5.8 of Pólya and Szegő [16. Using monotonicity, we also see that the limit as $r \rightarrow 1^{-}$of the right hand side of (1.6) exists, though it might be zero, as it is in the case $f(z)=(1-z) /(1+z)$. The case $p=2$ is a special case of the result of Laugesen and Morpurgo [11, Section 11] mentioned above. The case $p=1$ is a Schwarz Lemma for torsional rigidity, which answers a question left open in [4].

One can interpret these variations on the Schwarz Lemma as dynamic isoperimetric inequalities. As $r$ increases, geometric quantities such as diameter and area of $f(r \mathbb{D})$ increase as well, and the results of [4] state they increase at least as rapidly as the case when $f(r \mathbb{D})$ is a disk. Moreover, the case of equality only occurs if $f(r \mathbb{D})$ is a disk with centre $f(0)$. Similarly, Theorem 1 states that as $r$ increases the quantity $\mathcal{C}_{p}(f(r \mathbb{D}))$ decreases at least as quickly as that of the disk $r \mathbb{D}$, with equality if and only if $f(r \mathbb{D})$ is a disk with centre $f(0)$. 


\subsection{A reverse Hölder inequality for the minimiser of $\mathcal{C}_{p}$}

Payne and Rayner [14] published a reverse Hölder inequality for the first Dirichlet eigenfunction $\phi$ of the Laplacian - the minimiser of $\mathcal{C}_{2}(D)$ - that they had discovered some years before. Since they 'saw at that time little use for the inequality' and hoped to establish analogous results in higher dimensions they did not publish their result until 1972 coinciding with the publication of work by Sperb [17] in which he made 'extensive use of this inequality'. The Payne-Rayner inequality for the first Dirichlet eigenfunction $\phi$ is

$$
\left(\int_{D} \phi d A\right)^{2} \geq \frac{4 \pi}{\lambda(D)} \int_{D} \phi^{2} d A
$$

Several extensions of the original equality have since been obtained, including extensions to higher dimensions by Payne and Rayner [15] and KohlerJobin [10], to equations more general than $\Delta u+\lambda u=0$ with Dirichlet boundary conditions as in Alvino, Ferrone and Trombetti [1], Chiti [7] and Mossino [13] and, most recently, to the setting of minimal surfaces by Wang and Xia [18]. We prove the following extension.

Theorem 2. Let $n \geq 3$ and let $\Sigma \subset \mathbb{R}^{n}$ be a compact, embedded, minimal surface with Lipschitz, weakly connected boundary $\partial \Sigma$. For $p \geq 1$ let $\mathcal{C}_{p}(\Sigma)$ be given by (1.3) and let $\phi$ be a minimiser for $\mathcal{C}_{p}(\Sigma)$. Then

$$
\left(\int_{\Sigma} \phi^{p-1} d A\right)^{2} \geq \frac{8 \pi}{p \mathcal{C}_{p}(\Sigma)}\left(\int_{\Sigma} \phi^{p} d A\right)^{2-2 / p}
$$

Equality holds in (1.8) if and only of $\Sigma$ is a flat disk in an affine plane.

We explain the relevant terminology for minimal surfaces, including the definition of a weakly connected boundary, at the beginning of Section 3 . Also, observe that Theorem 2 includes the setting when $\Sigma$ is a bounded planar domain as a special case.

The line of thought behind our work in [5] was to obtain results for $\mathcal{C}_{p}$ that would interpolate between the corresponding results for torsional rigidity when $p=1$ and principal frequency when $p=2$, with the goal of both unifying and generalising such results. It is instructive from this point of view to note that, in the case of a bounded planar domain, the inequality (1.8) reduces, of course, to the classical Payne-Rayner inequality when $p=2$, and becomes the Saint Venant inequality $2 \pi P(D) \leq A(D)^{2}$, where $A(D)$ is the area of $D$, when $p=1$. The Saint Venant inequality is the isoperimetric inequality for torsional rigidity and states that, among 
all domains in the plane of given area, a disk of that area has the largest torsional rigidity. It was first proved by Pólya in 1948.

We can rewrite (1.8) more geometrically by giving $\Sigma$ the (singular) conformal metric $\widetilde{d s}=|\nabla \phi| d s$, where $d s$ is the arc-length element on $\Sigma$ making its inclusion in Euclidean space an isometric embedding. The length $L$ of $\partial \Sigma$ in this metric is

$$
L=\int_{\partial \Sigma}|\nabla \phi| d s=-\int_{\partial \Sigma} \frac{\partial \phi}{\partial \eta} d s=-\int_{\Sigma} \Delta \phi d A=\Lambda \int_{\Sigma} \phi^{p-1} d A,
$$

where $\partial / \partial \eta$ denotes the outward normal derivative and we have used (1.4). By (1.5),

$$
L=\frac{\mathcal{C}_{p}(\Sigma)}{\left(\int_{\Sigma} \phi^{p} d A\right)^{(p-2) / p}} \int_{\Sigma} \phi^{p-1} d A .
$$

The area $A$ of the surface $\Sigma$ in this conformal metric is, using the fact that $\phi$ is a minimiser for $\mathcal{C}_{p}(\Sigma)$,

$$
A=\int_{\Sigma}|\nabla \phi|^{2} d A=\mathcal{C}_{p}(\Sigma)\left(\int_{\Sigma} \phi^{p} d A\right)^{2 / p} .
$$

In terms of the length $L$ of the boundary and the area $A$ of the surface with respect to the conformal metric induced by the length of the gradient of the minimiser $\phi$ of $\mathcal{C}_{p}(\Sigma)$, the inequality (1.8) becomes

$$
L^{2} \geq \frac{8 \pi}{p} A
$$

with equality if and only if $\Sigma$ is a flat disk in an affine plane. As previously pointed out by the authors [6], this inequality has, in the case $p=2$ of the first Dirichlet eigenfunction for the Laplacian, the same form as the classical isoperimetric inequality.

\subsection{Organisation of the remainder of this paper}

We prove Theorem 11 in Section 2 by a suitable adaptation of the method of Laugesen and Morpurgo [11, Section 11]. Next we prove Theorem 2 in Section [3, the proof being modelled on Payne and Rayner's original proof for the case $p=2$.

ACKNowledGements: We first learned about variations on Schwarz's Lemma from Pietro Poggi-Corradini during a Summer School in Conformal Geometry, Potential Theory, and Applications held at NUI Maynooth in June 
2009. We would like to thank Pietro for interesting discussions on the subject and the organizers of the conference for providing a stimulating venue for these discussions. Michiel van den Berg kindly told us about the PayneRayner inequality when we needed precisely this result in connection with a different proof (see [6]) of a Schwarz Lemma for principal frequency. T. C. is partially supported by the ESF as part of the 'Harmonic and Complex Analysis and Applications' programme, and J.R. is partially supported by the University of Cape Town Research Committee.

\section{Proof of a Schwarz Lemma for $\mathcal{C}_{p}$}

For $\zeta \in \mathbb{D}$, let $g_{\zeta}$ be the conformal map of the disk $\mathbb{D}$ defined by $g_{\zeta}(z)=$ $f(\zeta z) / \zeta$. Set $\Omega_{\zeta}=g_{\zeta}(\mathbb{D})$. Note that $\Omega_{\zeta}$ is a rotation of $\Omega_{|\zeta|}$ so that $\mathcal{C}_{p}\left(\Omega_{\zeta}\right)$ depends only on $|\zeta|$. Moreover, $\Omega_{r}=f(r \mathbb{D}) / r$ for $0<r<1$ so that, by the scaling law, $\mathcal{C}_{p}\left(\Omega_{r}\right)=r^{4 / p} \mathcal{C}_{p}(f(r \mathbb{D}))$.

Set

$$
\Psi(\zeta)=\frac{1}{\mathcal{C}_{p}\left(\Omega_{\zeta}\right)}, \quad \zeta \in \mathbb{D} .
$$

Following the method of Laugesen and Morpurgo, we show that $\Psi^{p / 2}$ is subharmonic in $\mathbb{D}$. The integral means of this function are therefore both increasing and log-convex (see [9, Theorem 2.12]). Since $\Psi^{p / 2}$ is a radial function, its integral mean over the circle centre 0 and radius $r$ equates to $\Psi^{p / 2}(r)$, for $0<r<1$, which is therefore both increasing and log-convex. It follows that $\Psi(r)$ is increasing, so that $\mathcal{C}_{p}\left(\Omega_{r}\right)$ is decreasing. If, in addition, $p \leq 2$ then $2 / p \geq 1$ so that the function $\Psi(r)$ is log-convex on $0<r<1$.

The proof will therefore be complete once we show that $\Psi^{p / 2}$ is subharmonic in the disk. First,

$$
\Psi^{p / 2}(\zeta)=\sup \left\{\int_{\Omega_{\zeta}} u^{p} d A: u \in L^{p}\left(\Omega_{\zeta}\right) \cap W_{0}^{1,2}\left(\Omega_{\zeta}\right), u \geq 0, \int_{\Omega_{\zeta}}|\nabla u|^{2} d A=1\right\} .
$$

Write $\phi_{\zeta}$ for the extremal function on $\Omega_{\zeta}$ and $\psi_{\zeta}=\phi_{\zeta} \circ g_{\zeta}$ on $\mathbb{D}$. Since $g_{\zeta}^{\prime}(z)=f^{\prime}(\zeta z), z \in \mathbb{D}$,

$$
\Psi^{p / 2}(\zeta)=\int_{\Omega_{\zeta}} \phi_{\zeta}^{p} d A=\int_{\mathbb{D}} \psi_{\zeta}^{p}(z)\left|f^{\prime}(\zeta z)\right|^{2} d A(z) .
$$

Also, $1=\int_{\Omega_{\zeta}}\left|\nabla \phi_{\zeta}\right|^{2} d A=\int_{\mathbb{D}}\left|\nabla \psi_{\zeta}\right|^{2} d A$. In fact, if $\psi$ is any function on the disk for which $\int_{\mathbb{D}}|\nabla \psi|^{2} d A=1$ and we set $\phi=\psi \circ g_{\zeta}^{-1}$ on $\Omega_{\zeta}$ then

$$
\int_{\Omega_{\zeta}}|\nabla \phi|^{2} d A=1 \text { and } \int_{\Omega_{\zeta}} \phi^{p} d A=\int_{\mathbb{D}} \psi^{p}(z)\left|f^{\prime}(\zeta z)\right|^{2} d A(z)
$$


It then follows from (2.1) and (2.2) that

$$
\int_{\mathbb{D}} \psi^{p}(z)\left|f^{\prime}(\zeta z)\right|^{2} d A(z) \leq \int_{\mathbb{D}} \psi_{\zeta}^{p}(z)\left|f^{\prime}(\zeta z)\right|^{2} d A(z)
$$

Fix $\zeta \in \mathbb{D}$ and let $0<\rho<1-|\zeta|$. First using (2.1), then using (2.3) for the first inequality and the subharmonicity of $\left|f^{\prime}\right|^{2}$ for the second,

$$
\begin{aligned}
\frac{1}{2 \pi} \int_{0}^{2 \pi} \Psi^{p / 2}\left(\zeta+\rho e^{i \theta}\right) d \theta & \\
& =\frac{1}{2 \pi} \int_{0}^{2 \pi}\left(\int_{\mathbb{D}} \psi_{\zeta+\rho e^{i \theta}}^{p}(z)\left|f^{\prime}\left(\left(\zeta+\rho e^{i \theta}\right) z\right)\right|^{2} d A(z)\right) d \theta \\
& \geq \frac{1}{2 \pi} \int_{0}^{2 \pi}\left(\int_{\mathbb{D}} \psi_{\zeta}^{p}(z)\left|f^{\prime}\left(\left(\zeta+\rho e^{i \theta}\right) z\right)\right|^{2} d A(z)\right) d \theta \\
& =\int_{\mathbb{D}} \psi_{\zeta}^{p}(z)\left(\frac{1}{2 \pi} \int_{0}^{2 \pi}\left|f^{\prime}\left(\left(\zeta+\rho e^{i \theta}\right) z\right)\right|^{2} d \theta\right) d A(z) \\
& \geq \int_{\mathbb{D}} \psi_{\zeta}^{p}(z)\left|f^{\prime}(\zeta z)\right|^{2} d A(z) \\
& =\Psi^{p / 2}(\zeta),
\end{aligned}
$$

thereby establishing the sub-mean value property. The last inequality is strict unless $f$ is linear, making $\Psi^{p / 2}$ strictly subharmonic if $f$ is not linear. As shown by Laugesen and Morpurgo [11, Page 104], if a function is strictly subharmonic in the disk then its integral means are strictly increasing and strictly log-convex. This establishes the equality statement of Theorem 1 .

\section{Proof of a Payne-Rayner inequality}

In this section we prove a generalised Payne-Rayner inequality on a minimal surface. Some preliminary comments are in order. We consider a compact, two-dimensional, minimal surface $\Sigma \hookrightarrow \mathbb{R}^{n}$, with the induced metric, where $\Sigma$ has a Lipschitz boundary $\partial \Sigma$. The fact that $\Sigma$ is minimal means it is a critical point of the area function for variations which fix $\partial \Sigma$, or, equivalently, that the restriction of the coordinate functions to $\Sigma$ are all harmonic. Using the classical tools of the Riemannian geometry of surfaces, we define the usual gradient, divergence, and Laplace-Beltrami operators on $\Sigma$, and also

$$
\mathcal{C}_{p}(\Sigma)=\inf \left\{\frac{\int_{\Sigma}|\nabla u|^{2} d A}{\left(\int_{\Sigma} u^{p} d A\right)^{2 / p}}: u \in W_{0}^{1,2}(\Sigma), u \not \equiv 0\right\}
$$


as before. The Sobolev embedding is still compact for $p \geq 1$, and so a minimiser $\phi$ exists and solves the boundary value problem

$$
\Delta_{\Sigma} \phi+\Lambda \phi^{p-1}=0,\left.\quad \phi\right|_{\partial \Sigma}=0
$$

where $\Lambda$ and $\mathcal{C}_{p}(\Sigma)$ are related as in (1.5).

$\mathrm{Li}$, Schoen, and Yau defined the notion of a weakly connected boundary $\partial \Sigma$ for a surface $\Sigma \subset \mathbb{R}^{n}$ in 12 . The boundary $\partial \Sigma$ is weakly connected if there exists a rectangular coordinate system $\left\{x_{1}, \ldots, x_{n}\right\}$ for $\mathbb{R}^{n}$ such that no coordinate hyperplane $\left\{x_{j}=\right.$ constant $\}$ separates $\partial \Sigma$. They prove that if $\Sigma$ is a compact minimal surface with Lipschitz, weakly connected boundary $\partial \Sigma$ then it satisfies the isoperimetric inequality $(L(\partial \Sigma))^{2} \geq 4 \pi A(\Sigma)$, with equality if and only if $\Sigma$ is a flat disk.

Let $\left\{x_{1}, \ldots, x_{n}\right\}$ be the usual rectangular coordinates on $\mathbb{R}^{n}$ and define the function

$$
f=\frac{1}{2}\left(x_{1}^{2}+\cdots+x_{n}^{2}\right)
$$

restricted to $\Sigma$. As a result of $\Sigma$ being minimal we have $\Delta_{\Sigma} f=2$.

Proof of Theorem Q. Let $\phi_{M}=\max \{\phi(p): p \in \Sigma\}$ and, for $0 \leq t \leq \phi_{M}$, define

$$
\Sigma(t)=\{p \in \Sigma: \phi(p) \geq t\}, \quad S(t)=\{p \in \Sigma: \phi(p)=t\} .
$$

By Sard's theorem, $S(t)=\partial \Sigma(t)$ for almost every value of $t$.

Now define

$$
H_{0}(t)=\int_{\Sigma(t)} \phi^{p-1} d A, \quad H_{1}(t)=-\frac{p}{2} \int_{\Sigma(t)} \phi^{p-1}\langle\nabla \phi, \nabla f\rangle d A .
$$

It will be useful to rewrite $H_{1}(t)$ as

$$
\begin{aligned}
H_{1}(t) & =-\frac{p}{2} \int_{\Sigma(t)} \phi^{p-1}\langle\nabla \phi, \nabla f\rangle d A=-\frac{1}{2} \int_{\Sigma(t)}\left\langle\nabla\left(\phi^{p}\right), \nabla f\right\rangle d A \\
& =\frac{1}{2} \int_{\Sigma(t)} \phi^{p} \Delta_{\Sigma} f d A-\frac{1}{2} \int_{\partial \Sigma(t)} \phi^{p} \frac{\partial f}{\partial \eta} d s \\
& =\int_{\Sigma(t)} \phi^{p} d A-\frac{1}{2} \int_{\partial \Sigma(t)} \phi^{p} \frac{\partial f}{\partial \eta} d s .
\end{aligned}
$$

By the coarea formula, we have

$$
H_{0}^{\prime}(t)=\frac{d}{d t}\left(\int_{t}^{\phi_{M}} \tau^{p-1} \int_{S(\tau)} \frac{d s}{|\nabla \phi|} d \tau\right)=-t^{p-1} \int_{S(t)} \frac{d s}{|\nabla \phi|} .
$$


Observe that $\eta=-\frac{\nabla \phi}{|\nabla \phi|}$ is the outward unit normal to $\Sigma(t)$. Hence, by the divergence theorem

$$
\begin{aligned}
\int_{S(t)}|\nabla \phi| d s & =-\int_{S(t)} \frac{1}{|\nabla \phi|}\langle\nabla \phi,-\nabla \phi\rangle d s=-\int_{\Sigma(t)} \Delta_{\Sigma}(\phi) d A \\
& =\Lambda \int_{\Sigma(t)} \phi^{p-1} d A=\Lambda H_{0}(t) .
\end{aligned}
$$

Let $l(t)$ be the length of $S(t)$ and let $A(t)$ be the area of $\Sigma(t)$. Then combining (3.3) with the isoperimetric inequality for minimal surfaces [12] and the Cauchy-Schwarz inequality we have

$$
4 \pi A(t) \leq l^{2}(t) \leq\left(\int_{S(t)}|\nabla \phi| d s\right)\left(\int_{S(t)} \frac{d s}{|\nabla \phi|}\right)=\Lambda H_{0}(t) \int_{S(t)} \frac{d s}{|\nabla \phi|},
$$

which we can rearrange to read

$$
\int_{S(t)} \frac{d s}{|\nabla \phi|} \geq \frac{4 \pi A(t)}{\Lambda H_{0}(t)}
$$

Now, combining (3.2) and (3.4) we have

$$
\left(H_{0}^{2}(t)\right)^{\prime}=2 H_{0}(t) H_{0}^{\prime}(t) \leq-\frac{8 \pi A(t) t^{p-1}}{\Lambda} .
$$

Next we compute

$$
\begin{aligned}
\frac{d H_{1}}{d t} & =-\frac{p}{2} \frac{d}{d t}\left(\int_{\tau}^{\phi_{M}} t^{p-1} \int_{S_{\tau}} \frac{\langle\nabla \phi, \nabla f\rangle}{|\nabla \phi|} d s d \tau\right) \\
& =-\frac{p t^{p-1}}{2} \int_{\Sigma(t)} \Delta_{\Sigma} f d A=-p t^{p-1} A(t) .
\end{aligned}
$$

Combining (3.5) and (3.6) we have

$$
\frac{d}{d t}\left[H_{0}^{2}(t)-\frac{8 \pi}{p \Lambda} H_{1}(t)\right] \leq 0 .
$$

We integrate this last inequality from $t=0$ to $t=\phi_{M}$ and use $H_{0}\left(\phi_{M}\right)=$ $0=H_{1}\left(\phi_{M}\right)$ to obtain

$$
H_{0}^{2}(0) \geq \frac{8 \pi}{p \Lambda} H_{1}(0) .
$$

However, the definition of $H_{0}$ and (3.1) tell us that

$$
H_{0}(0)=\int_{\Sigma} \phi^{p-1} d A, \quad H_{1}(0)=\int_{\Sigma} \phi^{p} d A
$$


and so, using (1.5), we have

$$
\begin{aligned}
\left(\int_{\Sigma} \phi^{p-1} d A\right)^{2} & \geq \frac{8 \pi}{p \Lambda} \int_{\Sigma} \phi^{p} d A=\frac{8 \pi}{p} \frac{\left(\int_{\Sigma} \phi^{p} d A\right)^{(p-2) / p}}{\mathcal{C}_{p}} \int_{\Sigma} \phi^{p} d A \\
& =\frac{8 \pi}{p \mathcal{C}_{p}}\left(\int_{\Sigma} \phi^{p} d A\right)^{2-2 / p}
\end{aligned}
$$

which is (1.8).

If we have equality in (1.8) then we must have $4 \pi A(t)=l^{2}(t)$ for almost every $t$, which, by Theorem 1 of [12] implies $\Sigma$ is a flat disk in a two dimensional affine plane.

\section{References}

[1] A. Alvino, V. Ferone and G. Trombetti, On the properties of some nonlinear eigenvalues. SIAM J. Math. Anal. 29 (1998), 437-451.

[2] M. van den Berg, Estimates for the torsion function and Sobolev constants. to appear in Potential Analysis.

[3] D. Betsakos, Geometric versions of Schwarz's Lemmma for quasiregular mapings. preprint.

[4] R. Burckel, D. Marshall, D. Minda, P. Poggi-Corradini, and T. Ransford. Area, capacity, and diameter versions of Schwarz's lemma. Conform. Geom. Dyn. 12 (2008), 133-151.

[5] T. Carroll and J. Ratzkin, Interpolating between torsional rigidity and principal frequency. J. Math. Anal. Appl. 379 (2011), 818-826.

[6] T. Carroll and J. Ratzkin, Isoperimetric inequalities and variations on Schwarz's lemma. arXiv:SP/1006.2310.

[7] G. Chiti, A reverse Hölder inequality for the eigenfunctions of linear second order elliptic operators. Z. Angew. Math. Phys. 33 (1982), $143-148$.

[8] O. Druet and E. Hebey, The AB program in geometric analysis: sharp Sobolev inequalities and related problems. Mem. Amer. Math. Soc. 160 (2002), viii +98 pages. 
[9] W.K. Hayman and P.B. Kennedy, Subharmonic Functions, Volume 1. London Mathematical Society Monographs, No. 9, Academic Press, London, 1976.

[10] M.-T. Kohler-Jobin, Sur la première fonction propre d'une membrane: une extension à $N$ dimensions de l'inégalité isopérimétrique de PayneRayner. Z. Angew. Math. Phys. 28 (1977), 1137-1140.

[11] R. Laugesen and C. Morpurgo, Extremals for eigenvalues of Laplacians under conformal mappings. J. Funct. Anal. 155 (1998), 64-108.

[12] P. Li, R. Schoen, and S.-T. Yau, On the isoperimetric inequality for minimal surfaces. Ann. Scuola Norm. Sup. Pisa 11 (1984), 237-244.

[13] J. Mossino, A generalization of the Payne-Rayner isoperimetric inequality. Boll. Un. Mat. Ital. A (6) 2 (1983), 335-342.

[14] L. Payne and M. Rayner, An isoperimetric inequality for the first eigenfunction in the fixed membrane problem. J. Angew. Math. Phys. 23 (1972), 13-15.

[15] L. Payne and M. Rayner, Some isoperimetric norm bounds for solutions of the Helmholtz equation. Z. Angew. Math. Phys. 24 (1973), 105-110.

[16] G. Pólya and G. Szegö, Isoperimetric Inequalities in Mathematical Physics. Princeton University Press (1951).

[17] R. Sperb, Untere und obere Schranken für den tiefsten Eigenwert der elastisch gestützten Membran. Z. Angew. Math. Phys. 23 (1972), 231244.

[18] Q. Wang and C. Xia, Isoperimetric bounds for the first eigenvalue of the Laplacian. Z. Angew. Math. Phys. 61 (2010), 171-175. 